\title{
Arctiinae (Lepidoptera, Arctiidae) ocorrentes em uma área de pastagem na Amazônia Oriental em Altamira, Pará, Brasil
}

\author{
Márcia Cristina DELFINA ${ }^{1}$, José Augusto TESTON ${ }^{2}$
}

\begin{abstract}
RESUMO
O presente estudo avaliou a fauna de Arctiinae numa área de pastagem na Amazônia Oriental em Altamira-Pará, por meio de armadilha luminosa, com duas capturas mensais noturnas a cada fase da lua nova, no período de dezembro de 2008 a novembro de 2010. Foram avaliados os seguintes parâmetros: riqueza, abundância, dominância, constância, índices de diversidade e uniformidade de Shannon (H’ e E') e Brillouin (H e E), dominância de Berger-Parker (BP). As estimativas de riqueza foram feitas através dos procedimentos não paramétricos, "Bootstrap", "Chao1", "Chao2", "Jackknife1", "Jackknife2", e "MichaelisMentem”. Foram capturados 910 exemplares pertencentes a 85 espécies de Arctiinae. Os valores dos parâmetros analisados para o período total foram: $\mathrm{H}^{\prime}=2,58, \mathrm{E}^{\prime}=0,581, \mathrm{H}=2,45, \mathrm{E}=0,576 \mathrm{e} \mathrm{BP}=0,433$. Para os anos, tanto a riqueza quanto a abundância foi maior em 2009-2010. A diversidade e uniformidade de Shannon e Brillouin foram maiores para o ano de 2008-2009. Os estimadores previram um aumento entre 18,8\% e 85,9 \% na riqueza de espécies.
\end{abstract}

PALAVRAS-CHAVE: Biodiversidade, Estrutura de comunidade, Mariposas, Noctuoidea

\section{Arctiinae (Lepidoptera, Arctiidae) occurring in a pasture area in eastern Amazon in Altamira, Pará, Brazil}

\section{ABSTRACT}

This study evaluated the Arctiinae fauna of a pasture area in Altamira, Pará, eastern Amazon, using light traps. The moths were captured at every new moon phase, two nights per phase, from December 2008 to November 2010. We evaluated the following parameters: richness, abundance, dominance, constancy, diversity indexes and evenness (Shannon H' and E'; Brillouin H and E), and Berger-Parker dominance (BP). Richness estimates were made by nonparametric procedures: Bootstrap, Chao1, Chao2, Jackknife1, Jackknife2 and Michaelis-Mentem. We captured a total of 910 specimens belonging to 85 species of Arctiinae. Values of parameters for the total period were: $\mathrm{H}^{\prime}=2.58, \mathrm{E}^{\prime}=0.581, \mathrm{H}=2.45, \mathrm{E}=0.576$ and $\mathrm{BP}=0.433$. Both richness and abundance were higher in 2009-2010, while diversity and Shannon and Brillouin evenness were higher for the year 2008-2009. Estimators predicted an increase between $18.8 \%$ to $85.9 \%$ in species richness.

KEYWORDS: Biodiversity, Community structure, Noctuoidea,Tiger-moths

\footnotetext{
1 Universidade Federal do Pará - UFPA, Faculdade de Ciências Biológicas, Rua Coronel José Porfírio, 2515 - Bairro São Sebastião - $68372-040$ - Altamira, PA. E-mail: mc.delfino@hotmail.com

${ }^{2}$ Universidade Federal do Oeste do Pará - UFOPA, Programa de Pós-Graduação em Recursos Naturais da Amazônia (PGRNA) e Programa de Ciências Biológicas, Av. Marechal Rondon, s/n - Bairro Caranazal -68040-070 - Santarém, PA. E-mail: jateston@ufpa.br
} 


\section{INTRODUÇÃO}

As florestas primárias são convertidas em áreas para agricultura que agrupa áreas de culturas de ciclo longo e curto, agropecuária extensiva e intensiva, algumas dessas ficando abandonadas e transformando-se em florestas secundárias recentes, antigas e/ou capoeiras. Em média, cerca de 60\% da cobertura vegetal original da regiâo da Transamazônica e médio Xingu, já foram transformadas para uso agropecuário (Salomão et al. 2007).

Na Amazônia, o Pará é o estado em que a pecuária é mais importante, com o quinto rebanho bovino brasileiro de mais de 17 milhōes de cabeças, e extraordinário crescimento anual de cerca de $16 \%$. A pecuária é a principal atividade econômica em $51 \%$ dos municípios paraenses. (Minervino et al. 2008).

A intensa substituição das florestas pela formação de pastagem para a pecuária leva a uma enorme perda da diversidade de insetos. A grande maioria das espécies que habitam as florestas tropicais é susceptível ao processo de extinçáo, visto que estas ocorrem em baixas populaçóes (Thomazini eThomazini 2000).

O município de Altamira sofre uma considerável mudança na sua cobertura florestal original, devido à ação antrópica presente na zona de influência da rodovia transamazônica (BR 230) e suas transversais, cuja ocupação humana tem sido fortemente induzida por projetos de colonização agrária nos últimos 30 anos (Salomão et al. 2007).

Atualmente como o projeto de AHE Belo Monte no rio Xingu, aumenta ainda mais a preocupação com as espécies existentes nesse local, dessa forma, torna-se urgente à necessidade de analisar a biodiversidade dessa regiáo, promovendo o levantamento, identificaçáo e o registro das espécies (Teston e Delfina 2010).

Acredita-se que, se encontre algo em torno de $500 \mathrm{mil}$ espécies de Lepidoptera em todo mundo. No Brasil, são conhecidas quase 26 mil espécies de lepidópteros, quase metade das espécies da regiáo Neotropical (Duarte et al. 2012). No estado do Pará já há o registro de 497 espécies (Teston e Delfina 2010; Teston etal. 2012).

Mesmo existindo algumas espécies de hábito diurno, os arctiíneos constituem um dos grupos de lepidópteros noturnos mais utilizados como bioindicadores no monitoramento de ecossistemas (Hilty e Merenlender 2000). No Brasil, a fauna de lepidópteros noturnos tem sido estudada com armadilhas luminosas, especialmente na determinaçáo de índices faunísticos e distribuição desses organismos (Teston et al. 2006).

Este trabalho analisou a fauna de lepidópteros da subfamília Arctiinae noturnos, quanto a sua riqueza, abundância, e diversidade durante o período de dezembro de 2008 a novembro de 2010, numa área de pastagem na Amazônia Oriental no município de Altamira, Pará, Brasil.

\section{MATERIAL E MÉTODOS}

As atividades de coletas, realizadas durante duas noites a cada período de lua nova, devido esta proporcionar noites mais escuras e dessa forma não interferindo na captura, entre dezembro de 2008 a novembro de 2010, totalizando 46 amostragens (no mês de março de 2010 não houve capturas, devido a problema nos equipamentos de coleta), no sítio Vista Alegre (0307'22”S; 52¹5'17”W, altitude $130 \mathrm{~m}$ ), localizado no município de Altamira, Pará. A área de estudo apresenta forte ação antrópica e é constituída por pastagens e pomar com aproximadamente 28 anos de uso (Teston e Delfina 2010).

$\mathrm{Na}$ regiáo do estudo o período mais chuvoso estendese de dezembro a maio, e o menos chuvoso de junho a novembro (Moraes et al. 2005). Segundo as informaçóes obtidas na Estação de Meteorologia de Altamira, junto ao $2^{\circ}$ Distrito de Meteorologia (DISME) do Instituto Nacional de Meteorologia (INMET), durante o período de amostragem, foi apurada uma temperatura média de $28,4^{\circ} \mathrm{C}$ e pluviosidade total de $3.605,7 \mathrm{~mm}$, sendo no período mais chuvoso 27,4 ${ }^{\circ} \mathrm{C}$ e $2.938,7 \mathrm{~mm}$ e, no menos chuvoso $29,3^{\circ} \mathrm{C}$ e $667 \mathrm{~mm}$ de temperatura média e pluviosidade, respectivamente.

Para a captura dos arctíneos foi utilizada uma armadilha luminosa EMATER/RS, modelo "Santa Rosa” (Kober 1982) adaptada, possuindo um tronco de cone plástico com maior diâmetro de $32 \mathrm{~cm}$ e menor de $16 \mathrm{~cm}$, ao qual está acoplado um balde plástico com capacidade de 3,5 litros, onde são colocados 2 litros de álcool $92^{\circ} \mathrm{GL}$ e, equipada com lâmpada fluorescente ultravioleta F15 T8 BLB (Xelux, China) cuja luz possui comprimento de onda que varia de 290 a 450 e pico ao redor de 340 nanômetros. A armadilha foi instalada a uma altura de 2 metros do solo, acionada por bateria $12 \mathrm{~V}$, ao anoitecer $(18 \mathrm{~h})$ e desligada na manhá seguinte $(6 \mathrm{~h})$.

A triagem, identificação e contagem de todos arctíneos coletados, foram realizadas no Laboratório Integrado de Biologia e Educação Ambiental (LIBEA) da Faculdade de Ciências Biológicas do Campus Universitário de Altamira da Universidade Federal do Pará (UFPA). O processo de identificação, o posicionamento sistemático adotado e a distribuição das espécies dentro dos gêneros e tribos seguem Teston e Corseuil (2002; 2003a; b; 2004). Parte dos espécimes coletados foram preparado em alfinetes entomológicos e acondicionado em gavetas entomológicas, e a outra parte está conservada em envelopes entomológicos. O material testemunha (coleção de referência) encontra-se depositado na Coleção Entomológica do Museu de Zoologia (MZSTM) do Programa de Biologia da Universidade Federal do Oeste do Pará (UFOPA). 
Foram utilizados os parâmetros: riqueza $(S)$, abundância $(\mathrm{N})$ e índices de diversidade de Brillouin $(\mathrm{H})$ e Shannon ( $\left.\mathrm{H}^{\prime}\right)$, cujos valores encontrados foram comparados pelo teste " $\mathrm{t}$ " de Student, que se faz necessário, pois este índice assume que a amostra é uma parte da populaçáo entấo, para podermos compará-lo entre os períodos devemos realizar o teste de significância estatística. Isto não é necessário para o índice de Brillouin, devido a ele admitir que a amostra é a própria população entâo, o valor em si já é diferente. Uniformidade de Shannon (E'), de Brillouin (E) e dominância de BergerParker (BP) conforme (Magurran 2004), calculados para os períodos de mais e menos chuva e total. Foi avaliada também a similaridade entre os meses, períodos e anos de coleta através do índice de Bray-Curtis pelo método UPGMA, utilizando o software PAST versão 2.06 (Hammer et al. 2001). As estimativas de riqueza de espécies, para os períodos de mais e menos chuva e total, foram realizadas com auxílio do programa de computador "EstimatesRichnessEstimator" (Colwell 2009), empregando os procedimentos "Bootstrap", "Chao 1", "Chao2", "Jackknife1", "Jackknife2" e "Michaelis-Mentem", utilizando 1.000 casualizaçóes com abundância de classes igual a 10 (Colwell e Coddington 1994). Demais cálculos e gráficos foram realizados através de planilha eletrônica.

As categorias de dominância foram calculadas e classificadas segundo Ott e Carvalho (2001), em: eudominante, presentes em mais de $10 \%$ da amostragem; dominante entre 5-10\%; subdominante entre 2-5\%; eventual entre $1-2 \%$; e rara em menos de $1 \%$. A constância das espécies foi calculada e sua classificação realizada segundo Bodenheimer referido por Silveira Neto et al. (1976), em constantes, onde as espécies estavam presentes em mais de $50 \%$ das coletas, acessórias, presentes em $25 \%$ a $50 \%$ e acidentais, presentes em menos de $25 \%$.

\section{RESULTADOS E DISCUSSÃO}

Foram coletados 910 espécimes $(\mathrm{N})$, distribuídos em 85 espécies (S), com representante para as seis tribos pertencentes à subfamília Arctiinae (Tabela 1 e Figura 1).

Das 85 espécies capturadas, $72(84,71 \%)$ ocorreram com menos de 10 espécimes e destas, 38 estão representadas por somente um espécime ("singletons") e 13 por dois ("doubletons"). Doze espécies (14,12\%) ocorreram com mais de 10 espécimes, destacando-se Dycladia lucetius (Stoll, 1781), que foi a mais abundante com 394 espécimes, na sequência veio Paracles sp. 2 com 119 espécimes (Tabela 1). O fato de encontrar muitas espécies com poucos indivíduos é um padrão para comunidades de Arctiidae na regiáo Neotropical e já foi encontrado em alguns estudos efetuados no Brasil (Teston e Corseuil 2004; Ferro e Diniz 2007; Teston et al. 2009).

$\mathrm{Na}$ tabela 2, sáo apresentados os valores de riqueza de espécies (S), número de espécimes (N), índices de diversidade

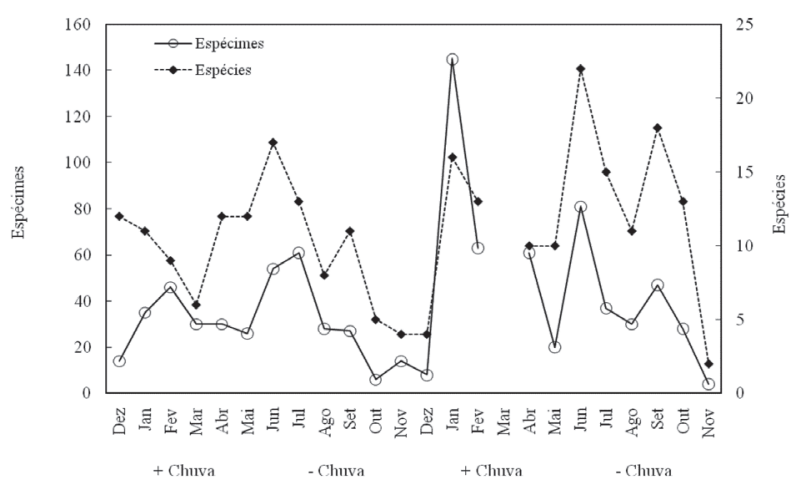

Figura 1 - Números de espécies e espécimes de Arctiinae (Lepidoptera, Arctiidae) capturados com armadilha luminosa em uma área de pastagem na Amazônia Oriental em Altamira, PA, Brasil, para os períodos mais (+ chuva) e menos chuvosos (- chuva), entre dezembro de 2008 a novembro de 2010.

de Shannon (H') e de Brillouin (H), uniformidade de Shannon (E') e Brillouin (E) e dominância de Berger-Parker (BP) por período (mais chuva e menos chuva) por ano e total.

Para os períodos, obteve-se maior valor de riqueza $(S)$ no período de menos chuva do ano de 2010 com 47 espécies, a maior abundância $(\mathrm{N})$ foi igual para o período de mais e menos chuva no ano de 2010 com 299 espécimes. Esta mesma tendência de capturar mais espécies no período de menos chuva também foi observado por Teston et al. (2012).O índice de diversidade de Shannon $\left(\mathrm{H}^{\prime}=2,79\right)$ e Brillouin $(\mathrm{H}=3,85)$ foi maior no período menos chuvoso de 2010. Este resultado também foi encontrado por (Teston et al. 2012) num fragmento de floresta neste mesmo município, tendo maior índice no período de menos chuva. A uniformidade de Shannon foi maior para o período de mais chuva de 2008$2009 \operatorname{com}\left(E^{\prime}=0,752\right)$, já a de Brillouin obteve $(E=0,748)$ também para o mesmo período. Para a dominância de BergerParker, o período com maior valor foi o mais chuvoso de 2010 com $(\mathrm{BP}=0,676)$. Ao contrário do que foi encontrado por (Teston e Delfina 2010), onde os valores foram maiores no período de menos chuva, exceto para a dominância.

Com relação aos índices, para os períodos anuais de amostragens, foram obtidos maior valor de riqueza $(S)$ para o período de dezembro de 2009 a novembro de 2010, com 67 espécies. A abundância (N) foi maior para o mesmo período com 528 espécimes. Os índices de diversidade de Shannon $\left(H^{\prime}=2,69\right)$, Brillouin $(H=2,51)$, e uniformidade de Shannon $\left(E^{\prime}=0,681\right)$ e Brillouin $(E=0,678)$ todos foram maiores para o mesmo período, dezembro de 2008 a novembro de 2009. Já na dominância de Berger-Parker $(\mathrm{BP}=0,509)$ o resultado foi maior para o período de dezembro de 2009 a novembro de 2010. Percebemos que para os períodos anuais a riqueza, abundância e dominância foram superiores para o segundo ano de amostragem. Já os índices de diversidade e uniformidade 
Tabela 1 - Número de espécimes, dominância ( $E$ = eudominante; $D=$ dominante; $S=$ subdominante; $E V=$ eventual; e $R=$ rara)e constância ( $A=$ acidentais; $\mathrm{B}=$ acessórias; e $\mathrm{C}=$ constantes) para Arctiinae (Lepidoptera, Arctiidae) capturados com armadilha luminosa em uma área de pastagem na Amazônia Oriental em Altamira, PA, Brasil, no período de dezembro de 2008 a novembro de 2010.

\begin{tabular}{|c|c|c|c|c|c|c|c|c|c|c|c|c|c|c|c|c|c|c|c|c|c|}
\hline & 2008 & & & & & & 2009 & & & & & & & & & 010 & & & & & \\
\hline Tribos / Espécies & $\mathrm{D}$ & $\mathrm{J}$ & $\mathrm{F}$ & M & A & M J & $\mathrm{J} J$ & A & S & $0 \mathrm{~N}$ & V D & $J$ & $\mathrm{~F} M$ & A & M & $J$ & J & A & $S C$ & $0 \mathrm{~N}$ & V Total \\
\hline \multicolumn{22}{|l|}{ ARCTIINI } \\
\hline Isia alcumena (Berg, 1882) (R) (A) & & & & 1 & 1 & 1 & & & & & & & & & & & & 1 & & & 4 \\
\hline Hypercompe sp. (R) (A) & & & & & & & & & & & & & & & & & 1 & & & & 1 \\
\hline Paracles sp. 1 (R) (A) & 1 & & & 1 & & & & & & & & & & 1 & & 1 & & 1 & & & 5 \\
\hline Paracles sp. 2 (E) (B) & 3 & & 3 & 4 & 6 & 119 & 94 & 4 & & & & 18 & & 16 & & 38 & 2 & 1 & & & 119 \\
\hline Paracles laboulbeni (Bar, 1873) (S) (B) & 3 & 1 & & 1 & & 3 & & 2 & & 2 & & 5 & & 3 & & 2 & & & 1 & & 23 \\
\hline Virbia subapicalis (Walker, 1854) (EV) (A) & 4 & & 2 & & & & & & & 2 & 2 & 1 & & & & & & 4 & 5 & & 18 \\
\hline Virbia sp. (EV) (A) & 1 & 1 & & & 1 & & 1 & & 2 & & & & & & & & & & 2 & 1 & 9 \\
\hline \multicolumn{22}{|l|}{ CALLIMORPHINI } \\
\hline Utetheisa ornatrix (Linnaeus, 1758) ${ }^{(\mathrm{R})}(\mathrm{A})$ & & & & & & & & 1 & & & & & & & & & 1 & & & & 2 \\
\hline \multicolumn{22}{|l|}{ CTENUCHINI } \\
\hline Aclytia heber (Cramer, 1780) ${ }^{(\mathrm{R})(\mathrm{A})}$ & & & & & & & & & 1 & & & & 1 & & & & 1 & & & & 3 \\
\hline Aethria andromacha (Fabricius, 1775) ${ }^{(\mathrm{R})}$ (A) & & & & & & & 2 & & & & & & & & & & & & & & 2 \\
\hline Antichloris eriphia (Fabricius, 1776) ${ }^{(\mathrm{R})(\mathrm{A})}$ & & & & & & & & & & & & & & & & & & & 1 & & 1 \\
\hline Correbia lycoides (Walker, 1854) (R) (A) & & & & & & 1 & & & & & & 1 & & & & 1 & & & 2 & & 5 \\
\hline Correbidia calopteridia (Butler, 1878) (R) (A) & & & & & & & 1 & & & & & & & & & & 1 & & 1 & & 3 \\
\hline Correbidia sp. ${ }^{(\mathrm{R})(\mathrm{A})}$ & & & & & & 2 & & & 1 & & & & & & 1 & & & & 1 & & 5 \\
\hline Delphyre discalis (Druce, 1905) ${ }^{(\mathrm{R})(\mathrm{A})}$ & & & & & & & & & & & 1 & & 1 & & & & & & & & 2 \\
\hline Epidesma albicincta (Hampson, 1905) ${ }^{(\mathrm{R})}$ (A) & & & & & & & & & & & & & & & & & & & & 1 & 1 \\
\hline Epidesma aurimacula (Schaus, 1905) (R) (A) & & & & & & & & & & & & & & & & 1 & & & & & 1 \\
\hline Epidesma parva (Rothschild, 1912) (R) (A) & & & & & & & & & & & & & & & & & & & & 1 & 1 \\
\hline Episcepsis frances Dyar, 1910 (R) (A) & & & & & & & & & & & & & & & & & & 1 & & & 1 \\
\hline Episcepsis lenaeus (Cramer, 1780) ${ }^{(\mathrm{R})}$ (A) & & & & & & & & & 1 & & & 1 & & & & & & & & & 2 \\
\hline Eucereon aoris Möschler, 1877 (R) (A) & & & & & & & & & & & & & & & & & & & & 1 & 1 \\
\hline Eucereon balium Hampson, 1898 (R) (A) & & & & & & & & & & & & & 1 & & & 1 & & & & 1 & 3 \\
\hline Eucereon maia Druce, 1884 (R) (A) & 3 & & & & & & & & & & & 4 & & & & & & & & & 7 \\
\hline Eucereon obscurum (Möschler, 1872) (R) (A) & & & & & & & & & & & & & & & & & 1 & & & & 1 \\
\hline Euclera meones (Stoll, [1780]) (R) (A) & & & & & & & & & & & 1 & & & & & & & & & & 1 \\
\hline Heliura rhodophila (Walker, 1856) ${ }^{(\mathrm{R})}$ (A) & & & & & & & & & & & 1 & 1 & & & & & & & & & 2 \\
\hline Napata leucotela Butler, 1876 (S) (A) & & & & & & & & & 1 & & & & 1 & 4 & & 1 & 3 & 3 & 8 & & 21 \\
\hline Telioneura brevipennis Butler, 1877 (R) (A) & & & & & & & & & & 1 & & & & & & & & & & & 1 \\
\hline Xanthopleura perspicua (Walker, 1856) (R) (A) & & & 1 & & & & & & & & & & & & & & & & & & 1 \\
\hline \multicolumn{22}{|l|}{ EUCHROMIINI } \\
\hline Calonotos triplaga Hampson, 1909 (EV) (A) & 4 & 1 & & & & & & & 1 & & & 2 & 3 & & & & & & & & 11 \\
\hline Cosmosoma subflamma (Walker, 1854) ${ }^{(\mathrm{R})(\mathrm{A})}$ & & 1 & & & & & & & & & & & 1 & & & & & & & & 2 \\
\hline Cosmosoma consolatum (Walker, 1856) (R) (A) & & & & & & 1 & & & & & & & & & & & & & & & 1 \\
\hline Cosmosoma metallescens (Ménétriés, 1857) ${ }^{(\mathrm{R})(\mathrm{A})}$ & & & & & & & & & & & & & 1 & & & & & & & & 1 \\
\hline
\end{tabular}


Continuação - Tabela 1

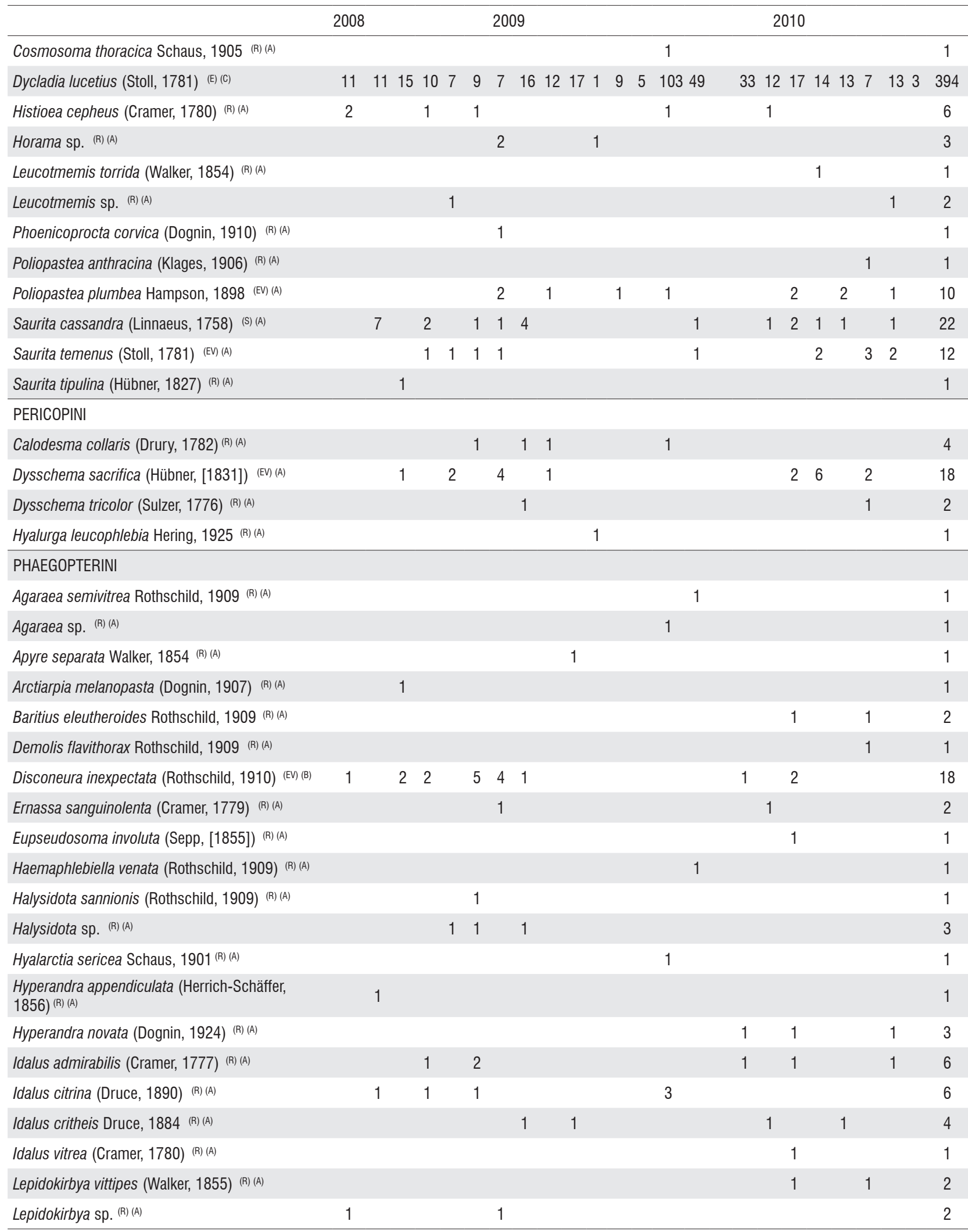


Continuação - Tabela 1

\begin{tabular}{|c|c|c|c|c|c|c|c|c|c|c|c|c|c|c|c|c|c|c|}
\hline & 2008 & & & & & 200 & & & & & & & & 2010 & & & & \\
\hline Leucanopsis sp. 1 (R) (A) & & & & 1 & 1 & 3 & & & & & & 1 & & 1 & & & & 7 \\
\hline Leucanopsis sp. 2 (R) (A) & & & & 2 & & 1 & 1 & & & & & & & & & & & 4 \\
\hline Lophocampa modesta (Kirby, 1892) (R) (A) & & & & & & & & & & & & & & 2 & & & & 2 \\
\hline Lophocampa sp. (EV) (A) & & & & & 4 & 3 & & & & & & & & 2 & & & & 9 \\
\hline Machaeraptenus ventralis Schaus, 1894 (R) (A) & & & & & & & & & & & & & & 1 & & & & 1 \\
\hline Melese asana Druce, 1884 (R) (A) & & & & 2 & & 1 & & & & & & & & & & & & 4 \\
\hline Melese incertus (Walker, 1855) (EV) (A) & 1 & & & & 1 & 1 & & & & & & & 1 & & 12 & 4 & & 12 \\
\hline Neonerita dorsipuncta Hampson, 1901 (R) (A) & 1 & & & & & & & & & & & & & & & & & 1 \\
\hline Ochrodota brunnescens Rothschild, 1909 (R) (A) & & & & & & & & & & 1 & & & & & & & & 1 \\
\hline Ordishia klagesi (Rothschild, 1910) (R) (A) & & & & & & & & & & & & & & & & & 1 & 1 \\
\hline Ormetica bonora (Schaus, 1905) (R) (A) & & & & & & & & & & & & & & 1 & & & & 1 \\
\hline Ormetica sypilus (Cramer, 1777) ${ }^{(\mathrm{R})}$ (A) & & & & & & & & & & 1 & & & & & & & & 1 \\
\hline Pareuchaetes aurata (Butler, 1875) (D) (B) & & 2 & 2 & 2 & 6 & 11 & 26 & 6 & 1 & & & & & & 1 & 5 & 3 & 65 \\
\hline Trichromia carinaria (Schaus, 1905) (R) (A) & & & & & & & & & & & & & & & 1 & & & 1 \\
\hline Viviennea moma (Schaus, 1905) (R) (A) & & & & & & & & & & & & & & 1 & & & & 1 \\
\hline TOTAIS & 36 & 2428 & 27 & 27 & 54 & 54 & 612 & 282 & 276 & 14 & 8145 & 63 & 61 & 208 & 3730 & 047 & 7284 & 910 \\
\hline
\end{tabular}

de Shannon e Brillouin foram maiores para o primeiro ano. Diferenciando do resultado obtido por Teston et al. (2012), onde todos os resultados foram maiores para o segundo ano, exceto dominância de Berger-Parker que foi mais baixa.

O índice de Shannon foi de $\left(H^{\prime}=2,58\right)$ e Brillouin $(\mathrm{H}=2,45)$, o de Berger-Parker $(\mathrm{BP}=0,433)$. A comparação dos índices de Shannon pelo teste "t" deu resultado não significativo para os períodos de mais e menos chuva (20082009) e menos chuva (2009-2010), mas entre os anos (20082009) e (2009-2010) houve diferença significativa.

$\mathrm{Na}$ análise de similaridade (Figura 2), segundo índice de Bray-Curtis, na primeira divisão o mês de outubro de 2009 (menos chuva) foi separado dos demais. Na segunda divisão os meses de dezembro de 2009 e novembro de 2010 (menos chuva) ficaram separados dos outros meses. Na terceira divisão os meses de janeiro, fevereiro e abril de 2010 (mais chuva) e junho também de 2010 (menos chuva) ficaram separados dos demais meses. Na quarta divisão o mês de setembro de 2010 (menos chuva) ficou separado dos outros meses. Na quinta divisão os meses de abril e maio de 2009 (mais chuva) e junho e julho também do mesmo ano (menos chuva) foram separados dos outros meses. Na sexta divisáo o mês de dezembro de 2008 (menos chuva) foi separado dos outros meses. Na sétima divisão o mês de novembro de 2009 (menos chuva) foi separado dos demais meses. Na oitava divisão os meses de janeiro de 2009 e maio de 2010 (mais chuva) e setembro de 2009 (menos chuva) ficaram separados dos demais meses. Já na nona divisão os meses de fevereiro e março de 2009 (mais

Tabela 2 -Valores de riqueza (S) e abundância (N) de espécies, índices de diversidade de Shannon ( $\left.H^{\prime}\right)^{\star}$ e de Brillouin $(H)^{\star}$, índices de uniformidade de Shannon $\left(E^{\prime}\right)$ e de Brillouin (E) e de dominância de Berger-Parker (BP) para Arctiinae (Lepidoptera, Arctiidae) capturados com armadilha luminosa em uma área de pastagem na Amazônia Oriental em Altamira, PA, Brasil, no período de dezembro de 2008 a novembro de 2010.

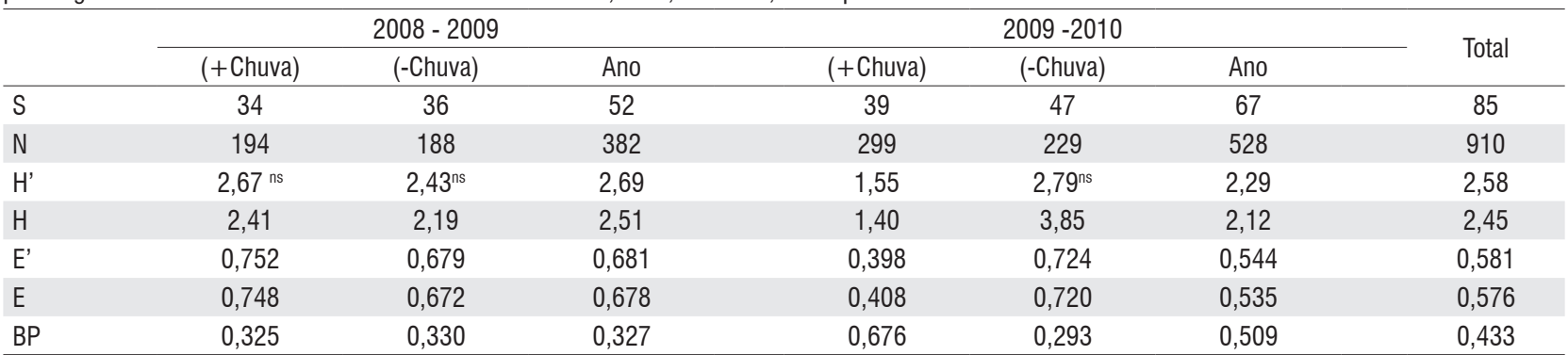

* (Logaritmo Natural); ns = nãosignificativoao nível de $5 \%$ pelo teste "t" 


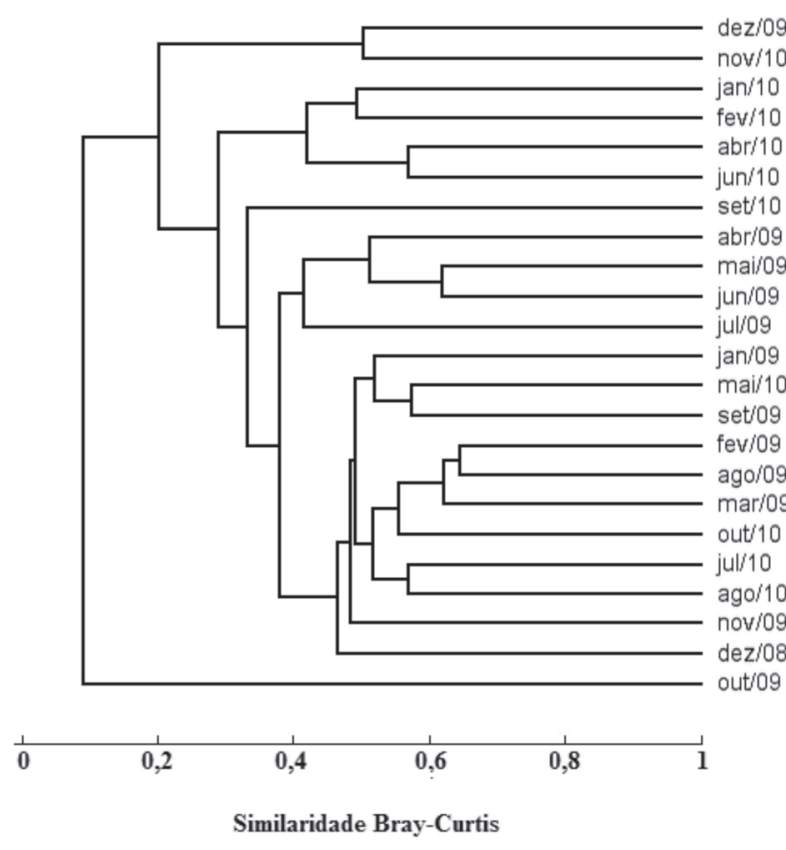

Figura 2 - Análise de agrupamento (UPGMA) baseado no índice de BrayCurtis para Arctiinae (Lepidoptera, Arctiidae) capturados com armadilha luminosa em uma área de pastagem na Amazônia Oriental em Altamira, PA, Brasil, no período de dezembro de 2008 a novembro de 2010.

chuvoso) agosto de 2009, julho, agosto e outubro de 2010 (menos chuva) foram separados dos demais meses.

Comparando os índices de similaridade entre os períodos percebemos que a maior similaridade foi encontrada entre os períodos de mais e menos chuva do ano de 2009 com $67 \%$. E o menor índice de similaridade foi entre os períodos de menos chuva de 2009 e de mais chuva de 2010, com 41\%. Já o índice de similaridade entre os anos foi de 58,9\%.

Para o período total, as estimativas de riqueza (Tabela 3) demonstram que podemos encontrar de 101 "Bootstrap" a 148 "Jackknife2" espécies.Este valor alto nas estimativas é devido ao elevado número de "Singletons" e "Doubletons" e demonstra que devemos encontrar muito mais espécies através da ampliação do período de amostragem, e indica ainda que a riqueza de Arctiinae em área alterada poderá ser maior do que no interior do fragmento de floresta no mesmo município (Teston et al. 2012), isto é devido, principalmente, em função do maior poder de atração proporcionada pela área alterada ser aberta, onde a luz da armadilha pode se difundir melhor no ambiente.

Na categoria de dominância (Tabela 1), foram registradas 70 espécies raras $(82,35 \%)$, nove eventuais $(10,59 \%)$, três subdominantes $(3,53 \%)$, uma dominante $(1,18 \%)$ e duas eudominantes $(2,35 \%)$. Entre as duas eudominantes,
Tabela 3 - Estimativas para Arctiinae (Lepidoptera, Arctiidae) capturados com armadilha luminosa em uma área de pastagem na Amazônia Oriental em Altamira, Pará, Brasil, no período de dezembro de 2008 a novembro de 2010. Números de amostras, espécies, números de espécies representadas por apenas um ("Singletons"), e dois exemplares (“Doubletons") e riqueza de espécies, segundo vários estimadores.

\begin{tabular}{|c|c|c|c|c|c|}
\hline & \multicolumn{2}{|c|}{ (2008-2009) } & \multicolumn{2}{|c|}{ (2009-2010) } & \multirow{2}{*}{ Total } \\
\hline & + Chuva & - Chuva & + Chuva & - Chuva & \\
\hline Amostras & 12 & 12 & 10 & 12 & 46 \\
\hline Espécies & 35 & 36 & 39 & 47 & 85 \\
\hline $\begin{array}{l}\text { Espécies } \\
\text { exclusivas }\end{array}$ & 11 & 18 & 26 & 23 & - \\
\hline "Singletons" & 12 & 18 & 26 & 23 & 38 \\
\hline "Doubletons" & 6 & 6 & 6 & 11 & 13 \\
\hline "Bootstrap" & 41 & 45 & 49 & 57 & 101 \\
\hline "Chao1" & 47 & 63 & 95 & 71 & 141 \\
\hline "Chao2" & 61 & 66 & 80 & 81 & 144 \\
\hline "Jackknife1" & 50 & 56 & 63 & 71 & 123 \\
\hline "Jackknife2" & 59 & 68 & 78 & 85 & 148 \\
\hline $\begin{array}{l}\text { "Michaelis- } \\
\text { Mentem" }\end{array}$ & 65 & 158 & 382 & 97 & 115 \\
\hline
\end{tabular}

novamente destaca-se Dycladia lucetius (Stoll, 1781), estando presente em 43 amostragens do total de 46, essa espécie também foi a mais capturada em pastagem em anos anteriores (Teston e Delfina 2010).

$\mathrm{Na}$ Constância das espécies (Tabela 1), foram obtidos os seguintes resultados, 80 espécies foram acidentais (94,12\%), quatro acessórias $(4,70 \%)$ e apenas uma espécie constante $(1,18 \%)$ que foi Dycladia lucetius (Stoll, 1781). Essa mesma espécie, também se destacou pela sua constância em área alterada, num período anterior (Teston e Delfina 2010).

A curva de rarefação de espécies (Figura 3) corrobora com o que foi apresentado pelos estimadores de riqueza, pois ela não apresenta uma tendência assíntota apontando que são necessárias mais amostras. Isso também pode ser verificado em outros estudos no município (Teston e Delfina 2010; Teston et al. 2012).

\section{CONCLUSÕES}

Destaca-se que a maioria das espécies ocorreu com frequências muito baixas com menos de dez exemplares. Dycladia lucetius (Stoll, 1781) foi a espécie mais abundante, dominante, constante e frequente, demonstrando ser mais tolerante a ambientes antrópicos. 


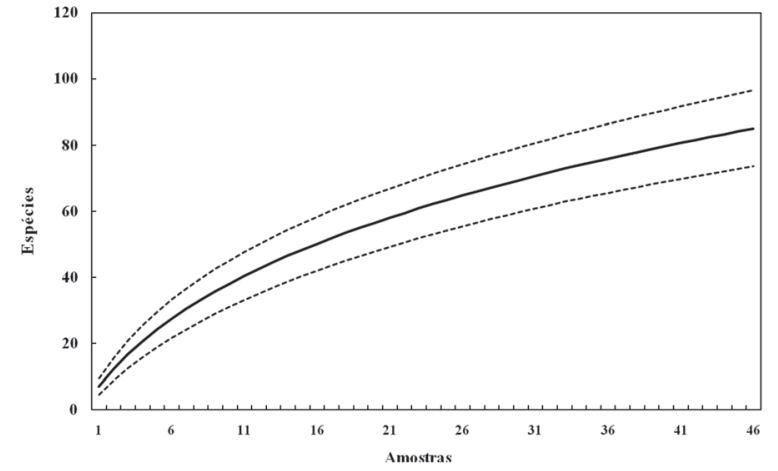

Figura 3 - Curva de rarefação de espécies de Arctiinae(Lepidoptera, Arctiidae) capturados com armadilha luminosa em uma área de pastagem na Amazônia Oriental em Altamira, PA, Brasil, no período de dezembro de 2008 a novembro de 2010, em relação ao esforço amostral, com os respectivos intervalos de confiança $95 \%$.

O estudo demonstrou que o período de menor precipitação pluviométrica foi o que obteve maior riqueza. Já a abundância não foi influenciada pela precipitaçáo. O período de menor precipitação de 2010 revelou melhores valores para a diversidade de Shannon e Brillouin, com relação à uniformidade o maior valor foi o de mais precipitação pluviométrica.

Para os anos tanto a riqueza quanto a abundância foi maior em 2010. A diversidade e uniformidade de Shannon e Brillouin foram maiores para o ano de 2009. A dominância de Berger-Parker obteve maior valor no ano de 2010, o que era esperado, pois esta é inversamente proporcional a diversidade e a uniformidade.

A análise de agrupamento não demonstrou haver uma diferença na similaridade entre os períodos de mais e menos chuva, no entanto, houve um agrupamento envolvendo quatro meses durante o período de menos chuva em 2009 e 2010.

As estimativas de riqueza demonstram que poderão ser encontras de $18,8 \%$ a 85,9 \% a maisde espécies na área de estudo com a ampliação do período de coletas.

\section{AGRADECIMENTOS}

Ao Sr. José Delfino Neto, por liberar a área para o estudo e auxiliar nas coletas. Estação de Meteorologia de Altamira (2。 DISME - INMET), pelas informaçóes meteorológicas. A Fundação de Amparo à Pesquisas do Estado do Pará (FAPESPA) por conceder auxílio financeiro para pesquisas com Arctiinae através do Edital Universal 2008 (Termo de Outorga e Aceitação de Auxílio n. ․ 138/2008), o qual permitiu a execução deste trabalho.

\section{BIBLIOGRAFIA CITADA}

Colwell, R.K. 2009. EstimateS: Statistical estimation of species richnessand shared species from samples. Version 8.2.0.User's Guide and application. (www.viceroy.eeb.uconn.edu/EstimateS). Acessoem 14/10/2011.

Colwell, R.K.; Coddington, J.A. 1994. Estimating terrestrial biodiversity through extrapolation.Philosophical Transaction of the Royal Society of London, Series B, 345: 101-118.

Duarte, M.; Marconato, G.; Specht, A.; Casagrande, M.M. 2012. Lepidoptera, p. 625-682. In: Rafael, J.A.; Melo, G.A.R.; Carvalho, C.J.B. de; Casari, S.A.; Constantino, R. (Eds.). Insetos do Brasil: diversidade e taxonomia. Holos, Ribeirão Preto (no prelo).

Ferro, V.G.; Diniz, I.R. 2007. Composição de espécies de Arctiidae (Lepidoptera) em áreas de Cerrado. Revista Brasileira de Zoologia, 24: 635-646.

Hammer, Ø.; Harper, D.A.T.; Ryan, P.D. 2001. PAST: Paleontological Statistics software package for education and data analysis. Palaeontologia Electronica, 4: 1-9.

Hilty, J.; Merenlender, A. 2000. Faunal indicator taxa selection for monitoring ecosystem health.Biological Conservation, 92: 185-197.

Kober, E.A.M. 1982. Armadilha luminosa: Informaçōes técnicas. EMATER/RS, Porto Alegre. 24 pp.

Magurran, A.E. 2004. Measuring Biological Diversity. Blackwell Publishing, Oxford. 260 pp.

Minervino, A.H.H.; Cardoso E. da C.; Ortolani E.L. 2008. Características do sistema produtivo da pecuária no município de Santarém, Pará. Acta Amazonica, 38: 11-16.

Moraes, B.C. de; Costa, J.M.N. da; Costa, A.L. da; Costa, M.H. 2005. Variação espacial e temporal da precipitação no estado do Pará. Acta Amazonica, 35: 207-214.

Ott, A.P.; Carvalho, G.S. 2001. Comunidade de cigarrinhas (Hemiptera: Auchenorrhyncha) de uma área de campo do município de Viamão, Rio Grande do Sul, Brasil. Neotropical Entomology, 30: 233-243.

Salomão, R. de P. ; Vieira, I.C.G.; Suemitsu, C.; Rosa, N. de A.; Almeida, S.S. de; Amaral, D.D. do; Menezes, M.P.M. de. 2007. As florestas de Belo Monte na grande curva do rio Xingu, Amazônia Oriental. Boletim Museu Paraense Emílio Goeldi, Ciências Naturais, 2: 57-153.

Silveira Neto, S.; Nakano, O.; Barbin, D.; Nova, N.A.V. 1976. Manual de ecologia dos insetos. Agronômica Ceres, Piracicaba. 419 pp.

Teston, J.A.; Corseuil, E. 2002. Arctiinae (Lepidoptera, Arctiidae) ocorrentes no Rio Grande do Sul, Brasil. Parte I. Pericopini. Biociências, 10: 261-268.

Teston, J.A.; Corseuil, E. 2003a. Arctiinae (Lepidoptera, Arctiidae) ocorrentes no Rio Grande do Sul, Brasil. Parte II. Arctiini, Callimorphini e Phaegopterini. Biociências, 11: 69-80. 
Teston, J.A.; Corseuil, E. 2003b. Arctiinae (Lepidoptera, Arctiidae) ocorrentes no Rio Grande do Sul, Brasil. Parte III. Ctenuchini e Euchromiini. Biociências, 11: 81-90.

Teston, J.A.; Corseuil, E. 2004. Diversidade de Arctiinae (Lepidoptera, Arctiidae) capturados com armadilha luminosa, em seis comunidades no Rio Grande do Sul, Brasil. Revista Brasileira de Entomologia, 48: 77-90.

Teston, J.A.; Delfina, M.C. 2010. Diversidade de Arctiinae (Lepidoptera, Arctiidae) em área alterada em Altamira, Amazônia Oriental, Pará, Brasil. Acta Amazonica, 40: 387-396.

Teston, J. A.; Novaes, J. B.; Almeida Júnior, J. O. B. 2012. Abundância, Composição e Diversidade de Arctiinae (Lepidoptera, Arctiidae) em um fragmento de floresta na Amazônia Oriental em Altamira, PA, Brasil. Acta Amazonica, 42: 19-28.
Teston, J.A.; Silveira, A.P.; Corseuil, E. 2009. Abundância, Composição e Diversidade de Arctiinae (Lepidoptera, Arctiidae) num fragmento de Mata Atlântica em Iraí, RS, Brasil. Revista Brasileira de Zoociências, 11: 65-72.

Teston, J.A.; Specht, A.; Di Mare, R. A.; Corseuil, E. 2006. Arctiinae (Lepidoptera, Arctiidae) coletados em unidades de conservação estaduais do Rio Grande do Sul, Brasil. Revista Brasileira de Entomologia, 50: 280-286.

Thomazini, M.J.; Thomazini, A.P. de B.W. 2000. A fragmentação florestal e a diversidade de insetos nas florestas tropicais úmidas. EMBRAPA Acre, Rio Branco. 21 pp(EMBRAPA Acre Documentos, 57).

Recebido em : 16-01-2012

Aceito em: 10-03-2012 
